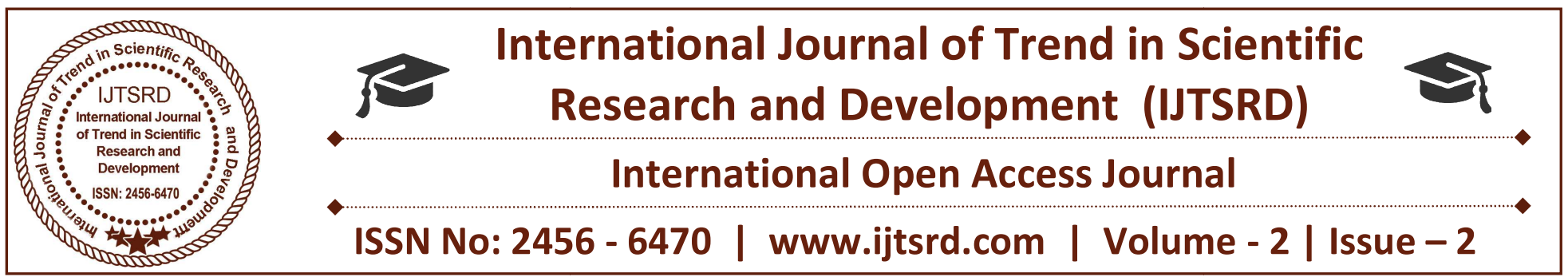

\title{
Injector Based Smart Vada Maker
}

Vishnu A

Assistant Professor, Department of Mechatronics Engineering, SNS College of Technology, Coimbatore, India
Arun Kumar K

UG Student, Department of Mechatronics Engineering, SNS College of Technology, Coimbatore, India
Praveen N

UG Student, Department of Mechatronics Engineering, SNS

College of Technology, Coimbatore, India
Sriram K

UG Student, Department of Mechatronics

Engineering, SNS College of

Technology, Coimbatore, India
Ashwin Ramnath S

UG Student, Department of Mechatronics Engineering, SNS College of

Technology, Coimbatore, India

\section{ABSTRACT}

A compact table-top automatic Vada making a machine for use at lunch counters, in-store bakery counters, small bakeries, and the like, where freshly cooked vada are sold in moderately large volume. The machine is particularly adaptable to varying volume requirements, being an adjustable crank mechanism employed in the system.

The machine includes a generally rectangular compact tank containing the cooking fat or oil which is heated by automatic thermostatically controlled means. A removable insert in the tanks defines a circuitous path along which the Vada flow in the course of being cooked. A removable vada extruding from the injector unit is mounted over one end of the tank to form and drop rings of raw dough into the oil. The Vada is moved along the surface of the cooking oil by a propulsion pump inducing a continuous flow of oil as the Vada is first cooked on one side, turned over automatically, cooked on the other side and discharged from the machine. The machine can be readily disassembled for easy cleaning.

Keywords: Automation, Injector unit, conveyor, Crank mechanism;

\section{Introduction}

The main concept is to introduce the standard kitchen is to provide the good infrastructure to enhance the benefits of Efficiency Safety and minimum wastage which is to achieve and ensuring quality and profitability. The Machine is designed in an Ergonomic manner and a compact design with speed preparation and Reliable performance.

The Machine includes a long relatively deep and narrow tank containing the cooking fat or oil which is heated by automatic thermostatically controlled Mean. The Vada extruding unit is mounted over one end of the tank to form and drop rings of raw dough into the oil. These are the manufacture of Premium quality raw material procured from reliable Source.

The Machine consists of two units one is the Injector unit and another one is frying unit, in the Normal Operation of the Vada Making machine. According to the present invention the tank is filled to the appropriate level with cooking oil and then heating element is turned on. The pump may also turn on to circulate the cooking medium to hasten to bring the oil to proper uniform temperature. 
The Extruder hopper system is filled with the mixer and variable speed extruder drive motor is set at the proper speed depending upon the desired volume of Vada to be produced in them.

A small Inexpensive vada fryer has the conveyor and separate extruder that driven from the same shaft without interference with each other.The sprocket for driving the elevator is recessed to receive rod from the conveyor.The method and apparatus for moving object to be fried through a hot frying liquid by producing intermittent waves and eddies in the liquid in desired direction of travel in straight line and also used the stepper motor to make the extruded vada from injector unit to flip and fry the thing on both side of them so that if we cause uniform frying in its process.

\section{Motivation of the project}

Our main motivation for doing this project is to improve automation in food technology, so we decide to develop a vada machine, in that we incorporate a compact tabletop machine. The Machine is designed in an Ergonomic manner and which is constructed based on compact shape and size of the machine. The Machine includes a long relatively deep and narrow tank containing the cooking fat or oil which is heated by automatic filled inside them.

- Comparatively, Cost Effective

- Hygienic way of Preparation

- Oil Consumption is Relatively Low

- Reduce floor Space

\section{Methodology}

Through literature reviews, we have formulated some of the existing methodologies and designed our system based on the difficulties faced by the existing authors.

\section{Existing Methodology}

There are two methods which are being the major disadvantage followed by the previously. They are

1. Manual Operated

2. No sensor is there to detect the oil at its boiling point;it just works on the principle of just to finish works.

The Flow Diagram of the existing methodology is shown below in Fig. 1

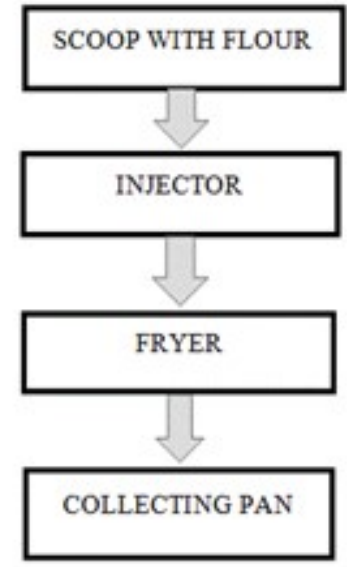

Fig. 1.Flow diagram of existing methodology

The Main disadvantage of the existing methodology is given below

- Floor space is large

- Relatively High cost

- Contain minimum number of automated system

- Time consumption of frying is high due to large space.

- Electricity waste occurs.

However, in our system, these disadvantages are overcome effectively.

\section{Proposed System}

The proposed system integrates all individual systems under one board. So that the overall cost of the system will be reduced efficiently.

The block diagram of the proposed system is shown in Fig. 2 .

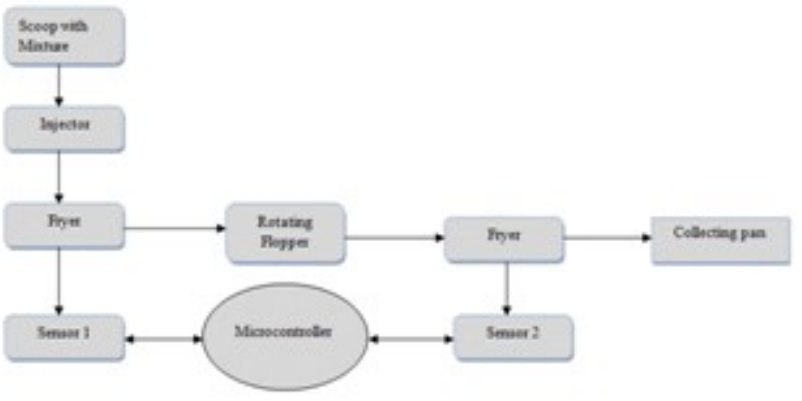

Fig.2.Flow diagram of proposed system

In the Proposed system, we incorporate several subsystems to make the production more efficient and effective in their performance. In this more over the 
system is completely an autonomous one, so that no manpower required of them.

The system comprises of sensors and microcontroller in that which the sensor sends the information to the controller and then it will do the action programmed in it, and then in frying pan is also an automated one because in that a rotating flipper is engaged in it, so that the vada will be fried in a proper manner.

Moreover, the system which works based on the injector unit in that it consists of hopper system so that it can able carry an amount of raw mixture and incorporate with anIn-Line crank mechanism (Vertical flow motion) using the $2 \mathrm{Hp}$ motor employed in the system.

Advantages of the proposed system are

- Integrated systems with Minimal Cost

- Predetermined shape and size

- Compact size so its oil consumption is low

- User-friendly Interface

- Improvised energy saving

The main disadvantages of this proposed system are given below

- Programming is more complex

- Electrical components must be handled with caution

- Time take to reach its boiling point is high.

\section{Procedure Flow}

The construction of the stepper motor is shown in Fig.4

The system follows three steps in automating the classroom. They are

1. Sensing

2. Transmission of data

3. Monitoring and Controlling

The sensor is the starting point of the process. Once the data is received the microcontroller does its work according to the program stored in it. The Flow diagram is shown in Fig.3

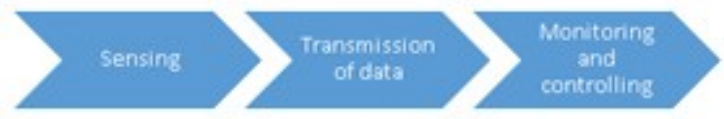

Fig.10 Procedure flow

\section{Components}

The selection of materials involves the study of their Characteristics, advantages, availability, cost, theuserfriendly property of components that we want to use. In our project, we select each and every component of study thoroughly about them. By proceeding like that only, we had done our selection.

The software and device chosen to programme the execution of our idea is MPLAB IDE and AT Mega Board.

The Software used to interface user and the machine.

The detailed description for selecting components is given below:

\section{A. Stepper Motor}

A stepper motor is a driver used to convert the electrical energy into discrete mechanical energy, the spindle shaft is rotated at the particular speed and it is used to rotate the flipper in the frying pan.

The reverse of this is the conversion of mechanical energy into electrical energy and is done by an electric generator, which has much in common with a motor. In this project, we use astepper motor for driving operation. The main purpose of stepper motor used in the project is for rotating the flipper enrolled in the frying pan system.

\section{(2)}
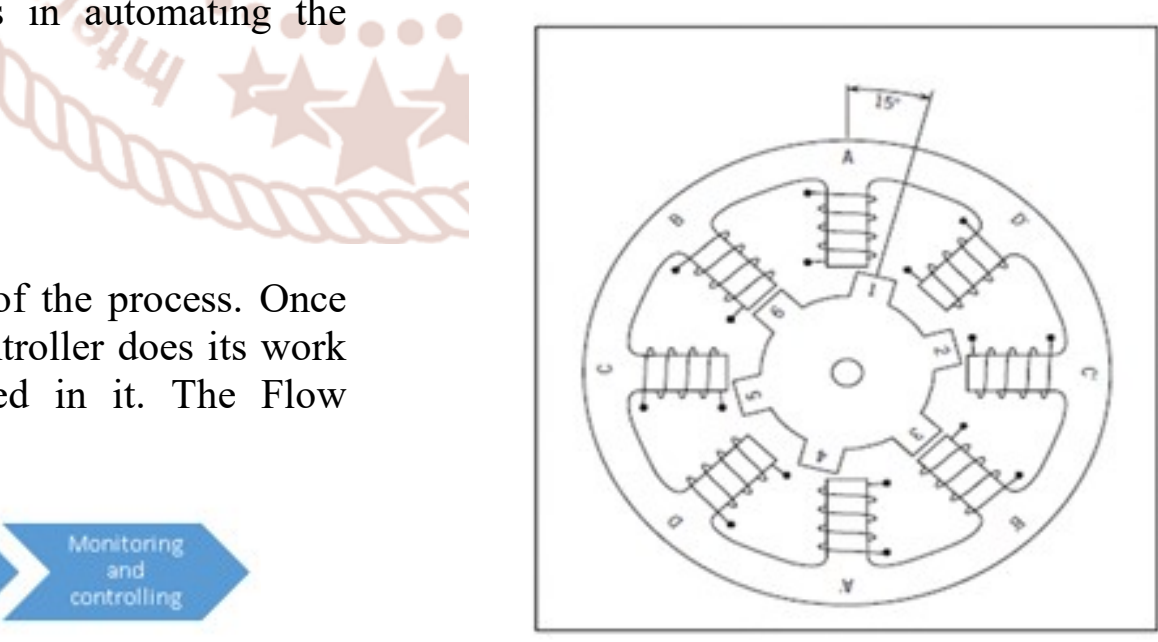

Fig.4. Construction of Stepper motor (Source: www.nptel.ni.in) 
The prime mover position would then be able to be instructed to move and hold at one of these means with no position sensor for criticism (an open-circle controller), as long as the engine is painstakingly estimated to the application in regard to torque and speed.

\section{B. Thermistor Sensor}

The sensoris sophisticated devices that are frequently used to detect and respond to electrical or optical signals. ASensor converts the physical parameter (for exampletemperature, blood pressure, humidity, speed, etc.) into a signal which can be measured electrically.

A Thermistor is sorts about resistor whose imperviousness may be reliant on temperature, that's only the tip of the iceberg something like that over standard resistors. The Thermistor is broadly utilized as inrush current limiter, temperature sensor, Selfresetting In current protectors What's more selfmanaging warming components.

The Thermistor Sensor working is shown in Fig. 5.

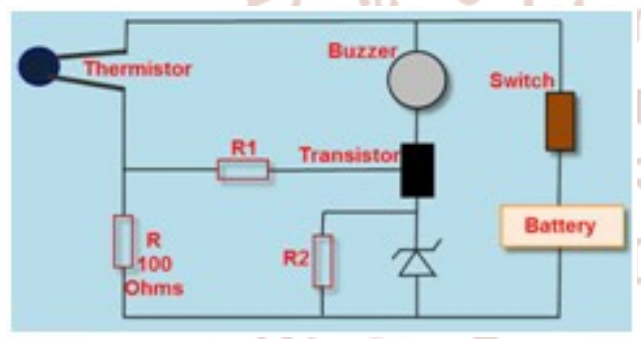

Fig.5. Thermistor Sensor working (source: www.elprocus.com)

\section{Injector}

The Injector is the major component of this project, moreover, the flour mixture is deposited in the scoop of this unit and the injector will extrude the mixture to the frying pan.

A removable dough extruder is movably mounted above the vessel adapted to be positioned over the upstream end of the circuitous flow path. A submerged pump will be arranged in the tank at the upstream limit of the stream way will circularize the cooking medium on gatherings give those transport frameworks to those vada. Thus Injector unit of Hopper system is shown in Fig.6.

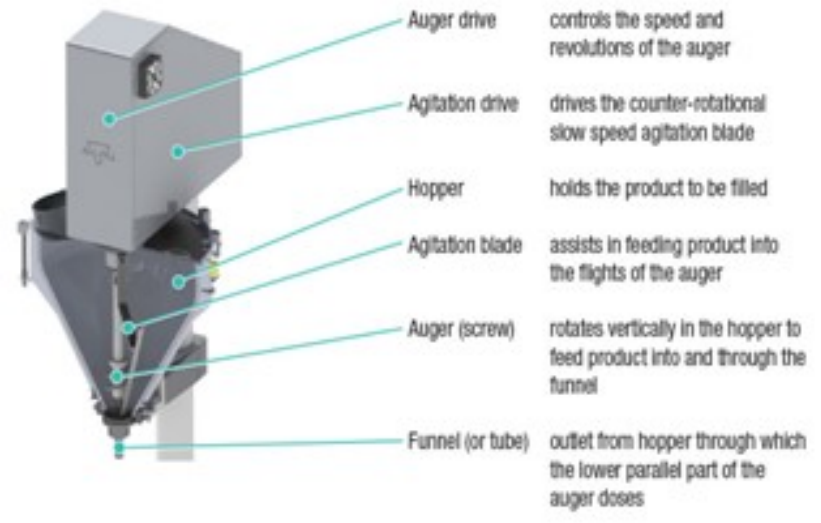

Fig 6: Injector unit working (source: www.google.com)
And the Major advantage of this system is completely automatic and so with run with 50 to $100 \mathrm{rpm}$. The injector unit worked based on In-line slider crank mechanism.

\section{Fryer}

The fryer is one of the important components of this project. Moreover, the word itself describes the use of this component, in this unit we build in the manner of little bit sliding form so that the extrude vada will move toward the outlet of the system. Thus fryer diagram is shown in Fig. 7.

\section{pmen}




\section{E. Microcontroller}

The microcontroller used in this project is ATMEGA $328 \mathrm{p}$.

The ATMEGA is a microcontroller family which consists of flash memory for program storage; it is similar to Arduino Uno R3. The ATMEGA controller which is a 28 pindual in-line package system (DIP). Moreover, it is used for Temperature monitoring and stepper motor control. The microcontroller diagram is shown in Fig.8.

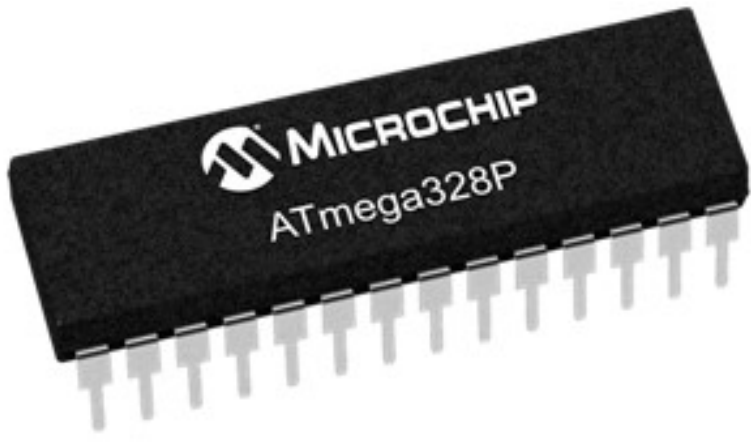

Fig 8: ATMEGA 328p Controller (source: www.microchip.com)

\section{Features:}

Table 1 describes the complete features of the ATMEGA microcontroller

\begin{tabular}{|l|l|}
\hline Feature & Specification \\
\hline Cpu type & 8 -Bit AVR \\
\hline Performance & $20 \mathrm{MHz}$ \\
\hline Flash memory & $32 \mathrm{~KB}$ \\
\hline SRAM & $2 \mathrm{~KB}$ \\
\hline EEPROM & $1 \mathrm{~KB}$ \\
\hline Pin count & $28 \mathrm{pin}$ PDIP, 32 pins TQFP \\
\hline $\begin{array}{l}\text { Maximum operating } \\
\text { frequency }\end{array}$ & $20 \mathrm{MHZ}$ \\
\hline $\begin{array}{l}\text { Number of touch } \\
\text { channel }\end{array}$ & 16 \\
\hline Maximum I/O pin & 26 \\
\hline
\end{tabular}

Table.1. Features of ATMEGA 328p microcontroller

\section{F. LCD 16X2 Matrix}

LCD matrices are great fun; used as a continuous temperature monitor. The temperature measurement is using LM35 an integrated sensor, the sensor converts the input signal to electrical signal by using ADC and the signal is displayed in the display. Sample LCD from Online is shown below in Fig 9.

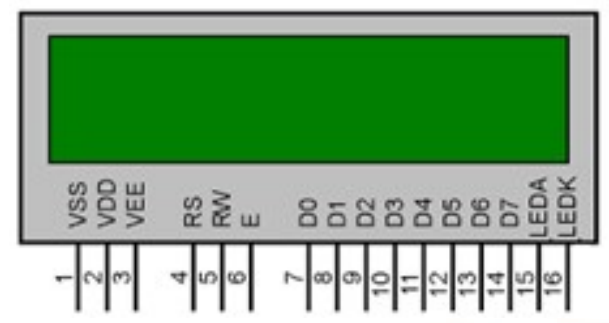

Fig 9.16X2 LCD Matrix

(Source: www.amazon.in)

A Scoop is a vast, pyramidal formed compartment utilized as a part of modern procedures to hold particulate mixture that has been gathered from theousted air. Scoop is typically introduced in gatherings to take into account a more prominent accumulation amount in them

Collecting pan is an element which collects fried vada from the fryer and dried it off. They are like storage system of extruded and fried vada in it.

The Table 2 which is listed below contains the overall components and their add-on features used in this project are shown in it.

\begin{tabular}{|l|l|}
\hline Component & Features \\
\hline Stepper motor & $10 \mathrm{~kg}-\mathrm{cm}, 5 \mathrm{Kg}-\mathrm{cm}$ \\
\hline Controller & $\begin{array}{l}\text { AT Mega 328p } \\
\text { Microcontroller 32 BIT - 28 } \\
\text { I/O PINS }\end{array}$ \\
\hline $\begin{array}{l}\text { Injector } \\
\text { In-line slider Crank } \\
\text { mechanism }\end{array}$ \\
\hline Power backup & 1 \\
\hline Thermistor Sensor & Generic k-type Sensor \\
\hline LCD Display & \begin{tabular}{l}
$16 X 2$ Matrix Display \\
\hline Cables
\end{tabular} \\
\hline $\begin{array}{l}\text { Framework and other } \\
\text { components }\end{array}$ & $\begin{array}{l}\text { Stainless steel, Aluminum } \\
\text { etc. }\end{array}$ \\
\hline
\end{tabular}

Table.2. Components used in the project

\section{Programming}

The programming is done in MPLAB IDE software. During programming, we have to keep in mind that all 
the systems should be automated and they should not get affected by the other parts of the program.

The Sample coding is given the future section.

Stepper Motor Control

void main() \{

TRISD $=0 \mathrm{~b} 0000000 ; / /$ PORT D as output port

PORTD $=0 \mathrm{~b} 1111111$

do

\{

PORTD $=0 \mathrm{~b} 00000011 ; \quad / /$ energizing two phases at a time

Delay_ms(500); // delay of $0.5 \mathrm{~s}$

PORTD = 0b00000110;

Delay_ms(500);

PORTD $=0 \mathrm{~b} 00001100$;

Delay_ms(500);

PORTD $=0 \mathrm{~b} 00001001$;

Delay_ms(500);

\} while(1); // loop executed infinite times \}

LCD Display

void READ_temp(void) \{

temp = ADC_Read $(0)$; temp $\quad=$ temp

temp $=$ temp $* 100$;

\}

void data_converstion(void)

\{

inttostr(temp,temper);

\}

void display1(void)

\{

lcd_out(1,1,"TEMPERATURE=");

lcd_out(1,13, Ltrim(temper));

Lcd_Chr_Cp(0xdf);

Lcd_Chr_Cp('C');

Lcd_Chr_Cp(' ');

\}

void main()

\{

ADC_Init();

Lcd_Init(); // Initialize LCD

Lcd_Cmd(_LCD_CLEAR); // Clear display

lcd_cmd(_LCD_CURSOR_OFF);
lcd_out(1,4,"DIGITAL TEMPERATURE");

lcd_out(2,6,"SENSOR");

delay_ms(1000);

Lcd_Cmd(_LCD_CLEAR); // Clear display while(1)

\{

READ_temp();

data_converstion();

display1();

\footnotetext{
\}
}

\section{Outcome expected}

There are two types of outcomes expected from this project. We have collected some data from the electric department and our group to achieve the required results.

\section{A. Energy Saving}

The data from the electric department which controls the power house of the college. The data is then compared with our assumption. The model graph is shown below in Graph.1.

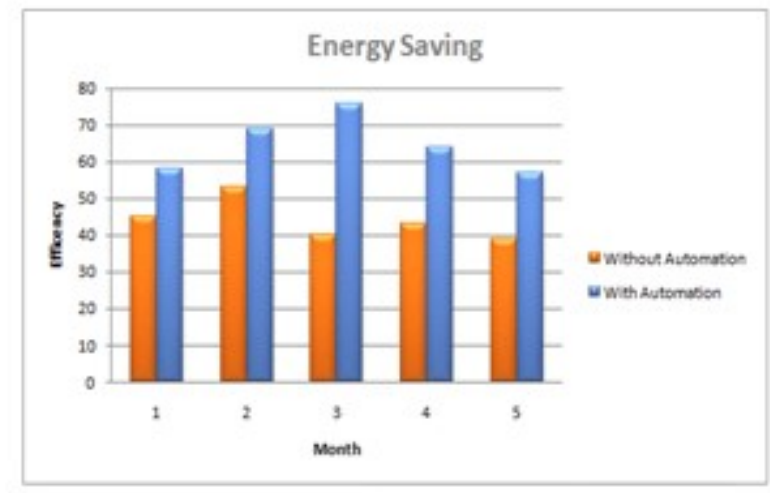

Graph 1: Assumed energy saving 


\section{B. Outcome Expected}

From the obtained data we had plotted a graph for the expected outcome in the below graph 2.

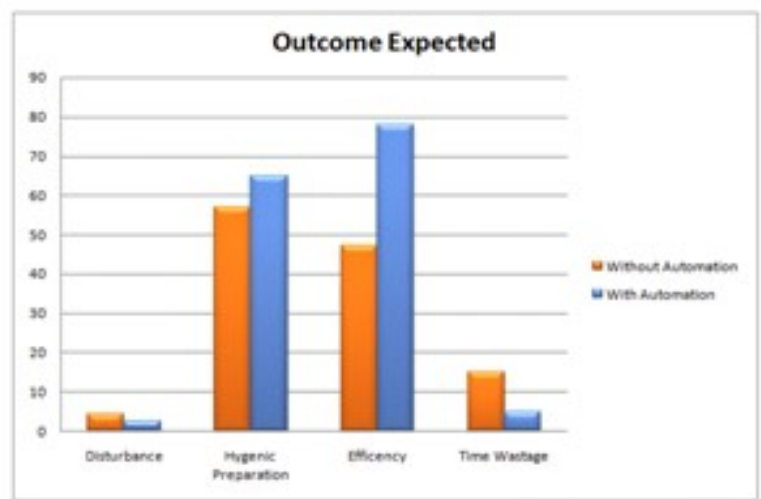

Graph 2: Outcome Expected

\section{Acknowledgment}

We take immense pleasure in expressing our humble note of gratitude to our project guide Mr.A.Vishnu Assistant Professor Department of / Mechatronics Engineering for his remarkable guidance in doing our project.

\section{References}

1. Journal on "design and development of an automatic dosa maker for indian households" by shaji K.S. in january 2016.

2. "Food guide lines and regulation" by FSMA(Food Safety Modernization Act) by U.S. Government on January 4,2011.

3. Paper on "LabVIEW Based Characterization and Optimization of Thermal Sensors" by NasrinAfsarimanesh and PathanZaheerAhamed at University of pune, India .

4. Debkumar Chakrabarti, (1997) Indian Anthropometric Dimensions, For Ergonomic Design Practice, NID India.

5. Paper on title "Stratification of thermoplastic olefins" by Pennington of USA on 1999.

6. Amos M. Fester "Automatic Doughnut Making Machine " united patent US4082033A in April 1976.

7. Herbert T Hunter "Doughnut machine" united patent US2067849A in January 1937.

8. William $\mathrm{O}$ Giles "Apparatus and process for continuously producing deep fat fried food products" united patent US2936698A in May 1958.

9. Anderson Edward M "Food cooking machine with control mechanism" united patent US4594941A in June 1986.

10. Philipsloan willy, LegrandJoseph S.Chen, "Energy efficient kitchens".Technology of electronics, Volume-4;issue-2, in 2009.

11. www.esp32.net

12. www.ebay.in

13. scholar.google.com

14. www.instructables.com

15. www.microchip.com 